\title{
Lois d'écoulement des laves torrentielles boueuses
}

\author{
Philippe Coussot \\ CEMAGREF, Division Protection contre les Erosions, \\ Domaine Universitaire, B.P. 76, \\ 38402 St-Martin-d'Hères, France \\ Tél. : 76762766
}

\begin{abstract}
L'étude des lois de comportement des laves torrentielles a fait l'objet de divers travaux récents. Coussot [1-2] a passé en revue et synthétisé les résultats obtenus. En première approximation on suppose que le matériau constitutif des laves torrentielles est un fluide homogène. Deux types de laves torrentielles ont été distingués [3-4] : les laves torrentielles granulaires au comportement « instable " encore trop mal compris ; les laves torrentielles boueuses qui contiennent une fraction argileuse non négligeable $(>10 \%)$ et ont une loi de comportement qui peut être correctement représentée par un modèle du type Herschel-Bulkley. On se propose ici de passer en revue les connaissances actuelles dans le domaine des lois d'écoulement des laves torrentielles boueuses qui sont les plus fréquentes dans les Alpes françaises [2].

Compte-tenu des hypothèses simplificatrices concernant le fluide constitutif des laves torrentielles [1], il s'agit ici d'étudier les lois d'écoulement des fluides de Herschel-Bulkley. Ce problème n'a pas fait l'objet d'études expérimentales ou théoriques systématiques. Les quelques travaux théoriques existants concernent les écoulements uniformes de fluides de Bingham ou d'autres fluides viscoplastiques simples [5-8] sur des plans inclinés. Le travail de Kozicki \& Tiu [9] fournit quant à lui une approche générale des écoulements uniformes de fluides non newtoniens dans des conduites de section quelconque mais n'a pas encore été validé expérimentalement. Plus généralement, concernant des écoulements à
\end{abstract}

surface libre laminaires de fluides à seuil, il n'existe pas à notre connaissance dans la littérature de comparaison des prédictions théoriques à partir de la loi de comportement du fluide avec les résultats expérimentaux. Les travaux expérimentaux se sont essentiellement attachés à définir la zone de transition entre les régimes laminaire et turbulent [10-13] ou à étudier les instabilités éventuelles de l'écoulement [14]. Nous nous baserons donc essentiellement sur les résultats de recherches développées récemment au CEMAGREF.

Il est important de préciser que les lois d'écoulement qui sont énoncées ci-après ne concernent que des écoulements permanents. Les écoulements transitoires qui correspondent en général mieux aux phénomènes naturels ne peuvent pas être décrits à l'aide de formules simples. Ces écoulements doivent être étudiés par d'autres moyens : modélisation numérique [15] ou étude sur modèle réduit [16].

L'objet de cet article est de fournir des formules utilisables en pratique pour décrire correctement les écoulements permanents de boue ou pour estimer rapidement mais approximativement les caractéristiques des écoulements de laves torrentielles transitoires.

Pour tous les développements théoriques qui suivent on fera les hypothèses suivantes :

- Le fluide considéré est homogène à tout point de vue (concentration, densité, loi de comportement).

- L'écoulement est laminaire. Cette hypothèse a été

\section{Debris flow dynamics}

Debris flows are large masses of water, clay, silt, sand, pebbles and boulders which sometimes flow in mountain streams after long or intense rains and can cause much damages to inhabited areas. Muddy debris flows which are the most common in France exhibit a Herschel-Bulkley behaviour. Here we present theoretical tools for predicting steady free surface debris flows.

We first show the fundamental feature of channelized debris flows: an unsheared zone (called plug) within the material. For flow on an infinitely wide plane one can easily compute flox characteristics. From these formulae we deduce an expression for the wall shear stress. We also propose other expressions, established empirically, which may be used for flow in rectangular or trapezoidal channels. We then show that global steady flow characteristics are similar to water flows (supercritical and subcritical regimes, hydraulic jump, rol waves). Nevertheless flow through singularities are somewhat different (dead zones formation). 
vérifiée a posteriori [17] en utilisant un critère de Reynolds généralisé (adapté aux fluides non newtoniens). Elle a aussi été vérifiée expérimentalement en laboratoire en comparant les prédictions théoriques et expérimentales concernant les hauteurs normales d'écoulement.

- Il n'y a pas en général de glissement à la paroi. Cette hypothèse a été vérifiée expérimentalement a posteriori [17] en comparant les résultats obtenus avec des parois de différentes rugosités.

Dans une première partie on présente les caractéristiques essentielles d'un écoulement uniforme à partir du cas particulier de l'écoulement sur un plan infiniment large qui possède une solution analytique simple. Dans la seconde partie on présente des expressions pour la contrainte moyenne à la paroi dans le cas d'un canal de section rectangulaire ou trapézoïdale. On montre ensuite dans la troisième partie comment ces expressions peuvent être utilisées pour prédire les écoulements graduellement variés. Enfin dans la dernière partie on s'intéresse aux caractéristiques des écoulements au passage de singularités.

\section{Généralités}

\subsection{Loi de comportement}

Comme on l'a vu précédemment [1] la loi de comportement des laves torrentielles boueuses peut être représentée correctement par un modèle de Herschel \& Bulkley. En outre, de façon à simplifier le traitement théorique, on fait I'hypothèse que le coefficient $n$ peut être systématiquement supposé égal à $1 / 3$, sans que cela altère la qualité du calage du modèle sur les rhéogrammes expérimentaux [1]. La loi de comportement des laves torrentielles boueuses en cisaillement simple (type d'écoulement le plus courant lors d'écoulements à surface libre) s'exprime ainsi :

$$
\begin{array}{lll}
\tau=\tau_{c}+K \dot{\gamma}^{1 / 3} & \text { quand } & \dot{\gamma} \neq 0 \\
\tau \leqslant \tau_{c} & \text { quand } & \dot{\gamma}=0
\end{array}
$$

où $\tau$ est la contrainte tangentielle, $\dot{\gamma}$ le gradient de vitesse, et $\tau_{c}, K$ et $n$ sont des paramètres du fluide.

\subsection{Similitude}

Considérons un fluide ayant une loi de comportement du type (1) en écoulement dans un canal incliné (de pente $i)$ sous l'action de la gravité $(g)$. Alors les trois nombres adimensionnels qui gouvernent l'écoulement sont :

$$
\begin{gathered}
F=\frac{U}{\sqrt{g(\cos i) h}} ; \\
G=\frac{\rho g h(\sin i)}{\tau_{c}} ; \quad H_{b}=\frac{\tau_{c}}{K}\left(\frac{h}{U}\right)^{1 / 3}
\end{gathered}
$$

où $U$ est la vitesse moyenne du fluide dans une section en travers, $\rho$ la masse volumique du fluide, et $h$ une hauteur de référence. Pour des études sur modèle réduit en similitude, le respect de l'égalité de ces trois paramètres entre le prototype et la réalité est relativement simple à réaliser. En effet, si $\lambda$ est le rapport entre une longueur de référence du prototype et la longueur correspondante dans la réalité, on devra respecter, outre la similitude des conditions aux limites et initiales, les égalités suivantes :

$$
\tau_{c 2}=\tau_{c 1} \frac{\rho_{2}}{\rho_{1}} \lambda ; \quad K_{2}=K_{1} \frac{\rho_{2}}{\rho_{1}} \lambda^{7 / 6}
$$

entre les caractéristiques du fluide modèle et du fluide réel. Ceci est relativement facile à réaliser en utilisant comme fluide modèle un mélange boueux fin à une concentration solide adaptée pour que la réduction imposée sur les paramètres $K$ et $\tau_{c}$ soit globalement respectée.

\subsection{Caractéristiques générales des écoulements}

On se propose ici de mettre en valeur les caractéristiques principales des écoulements de laves torrentielles boueuses en examinant un cas particulier très représentatif. On considère un écoulement permanent uniforme sur un plan incliné infiniment large. Dans ce cas les équations du mouvement peuvent être résolues complètement et exactement [18]. On peut en déduire notamment la composante non nulle de la vitesse dans la direction de plus grande pente du plan et en un point du fluide situé à $y$ au-dessus du plan :

$$
\begin{aligned}
& u(y)=\frac{\alpha}{4}\left[y_{0}^{4}-\left(y_{0}-y\right)^{4}\right] \text { quand } y \leqslant y_{0} \\
& u(y)=u\left(y_{0}\right)=\frac{\alpha}{4} y_{0}^{4} \text { quand } h \geqslant y \geqslant y_{0}
\end{aligned}
$$

avec

$$
y_{0}=h-\frac{\tau_{c}}{\rho g(\sin i)} ; \quad \alpha=\left(\frac{\rho g \sin i}{K}\right)^{3} .
$$

Ce profil des vitesses très particulier est caractéristique des fluides à seuil: il existe une zone (d'épaisseur $y_{0}$ ) au contact de la paroi dans laquelle le fluide est cisaillé alors que le reste du fluide n'est pas cisaillé (cf. fig. l). En fait, dans cette seconde zone, tout se passe

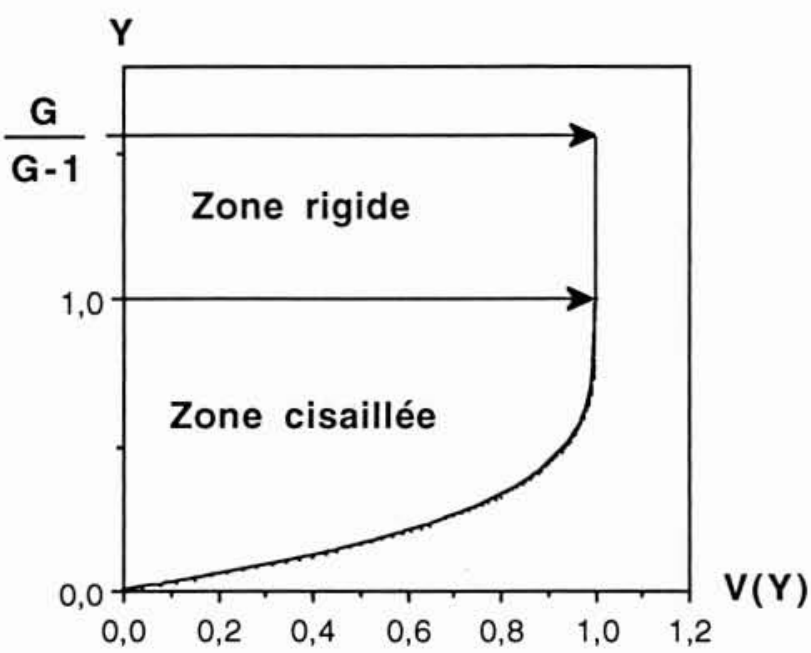

1. Distribution des vitesses au sein d'un écoulement permanent uniforme d'une lave torrentielle sur un plan incliné. 
comme si le fluide était rigide. On peut aussi remarquer que l'intensité du cisaillement augmente beaucoup lorsqu'on se rapproche de la paroi. Ces caractéristiques peuvent être globalement observées dans tous les écoulements lorsqu'on n'est pas trop loin des conditions du régime uniforme.

Enfin il est important de noter que, si :

$$
h<\frac{\tau_{c}}{\rho g(\sin i)}\left(=h_{0}\right)
$$

il n'y a pas écoulement. On voit ainsi apparaître une hauteur limite $\left(h_{0}\right)$ au-delà de laquelle un écoulement uniforme peut se produire et en deçà de laquelle le fluide est rigide (en régime permanent). Pour une hauteur donnée, on en déduit aussi facilement une pente critique (liée à la hauteur limite par (5)) au-delà de laquelle le fluide commence à s'écouler. Ces caractéristiques sont elles-aussi générales. Quel que soit le type d'écoulement considéré, il existe une pente et une hauteur limites d'écoulement. Ceci explique les lobes ou les zones d'arrêt de forte épaisseur que l'on peut observer dans la nature.

Enfin, on peut déduire directement de (4) le débit $q$ par unité de largeur dans une section en travers de l'écoulement :

$$
q=\frac{\alpha}{4} y_{0}^{4}\left[h-\frac{y_{0}}{5}\right] .
$$

La contrainte à la paroi est uniforme et s'écrit :

$$
\tau_{p}=\rho g(\sin i) h .
$$

\section{Ecoulement uniforme dans des canaux de section quelconque}

Dans le cas de l'écoulement uniforme sur un plan infiniment large, la hauteur normale peut être calculée à l'aide de la formule (6). La validité de ces calculs a été vérifiée lors d'expériences d'écoulements de boue dans des canaux rectangulaires (en similitude d'écoulements de laves torrentielles) [17]. Il a ainsi été montré que l'hypothèse du plan infiniment large conduit à des erreurs négligeables sur l'estimation de la hauteur normale tant que le rapport entre cette hauteur et la largeur du canal (L) est inférieur à 0,1 (cf. fig. 2).

Dans le cas de l'écoulement uniforme dans un canal de section quelconque, il n'est pas possible de résoudre de manière analytique simple les équations du mouvement. Il n'est donc pas possible d'établir une expression théorique pour le débit. Plutôt que d'établir empiriquement de telles expressions pour des canaux de diverses sections en travers, il s'avère plus utile de déterminer empiriquement des expressions de contraintes moyennes à la paroi. Ces expressions serviront à calculer la hauteur normale de l'écoulement uniforme et pourront être utilisées dans les calculs d'écoulements graduellement variés ou transitoires ou encore pour en déduire un critère de stabilité (cf. paragraphes suivants). Par exemple dans le cas de l'écoulement sur un plan infiniment large on peut

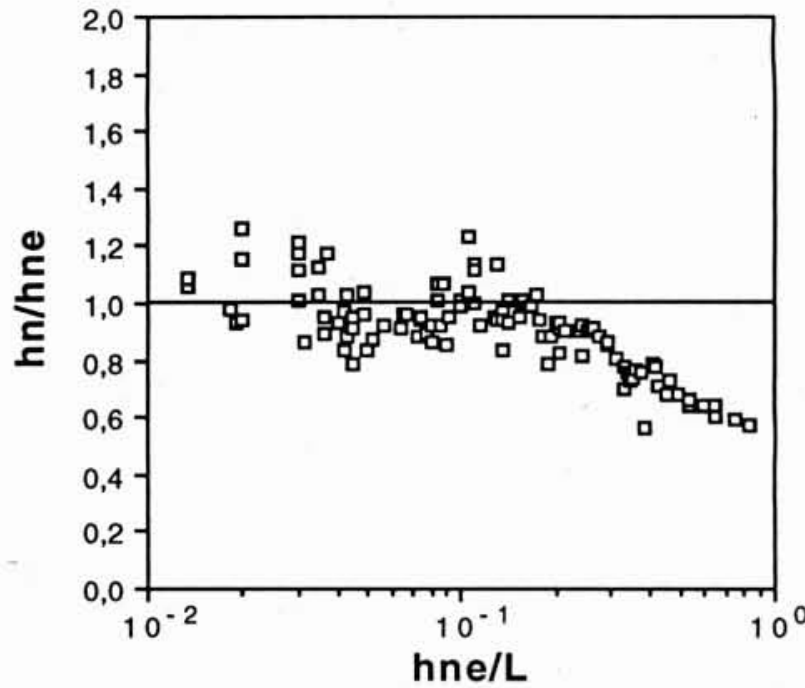

2. Comparaison hauteur normale expérimentale $\left(h_{n e}\right)$ - hauteur théorique $\left(h_{n}\right)$ (en utilisant la formule $\left.(6)\right)$ pour des écoulements uniformes en canal rectangulaire, en fonction du rapport $h_{n d} / L$ [17].

déduire de (6) et (7) l'expression approchée suivante pour la contrainte à la paroi :

$$
\tau_{p}=\tau_{c}\left(1+1,93\left(H_{b}\right)^{-0.9}\right) .
$$

Cette expression peut aussi être écrite sous forme adimensionnelle :

$$
G=\left(1+1,93\left(H_{b}\right)^{-0.9}\right) .
$$

Dans [17] des expressions semi-empiriques de contrainte à la paroi ont été établies. Systématiquement, il a été supposé que celles-ci pouvaient prendre la forme de relations entre $G, H_{b}$ et des paramètres de forme de l'écoulement [18]. De façon à généraliser (9), $G$ est alors le rapport entre la contrainte moyenne à la paroi et le seuil de contrainte du fluide. En revanche $H_{b}$ est toujours calculé en utilisant la hauteur maximum de fluide. Les expressions suivantes ont ainsi été établies :

$$
\tau_{p}=\tau_{c}\left(1+a\left(H_{b}\right)^{-0.9}\right)
$$

avec :

- Canal rectangulaire :

$$
a=1,93-0,43 \arctan \left[\left(\frac{10 h}{L}\right)^{20}\right] ; \frac{h}{L}<1(11)
$$

- Canal trapézoïdal (base $B$, pente des côtés : $45^{\circ}$ ) :

$$
a=1,93-0,6 \arctan \left[\left(\frac{0,4 h}{B}\right)^{20}\right] ; \frac{h}{B}<4 \text {. }
$$

Avec ces formules on peut calculer la hauteur normale d'écoulement (correspondant au régime uniforme) en utilisant l'autre expression de la contrainte moyenne à la paroi :

$$
\tau_{p}=\rho g R \sin i .
$$




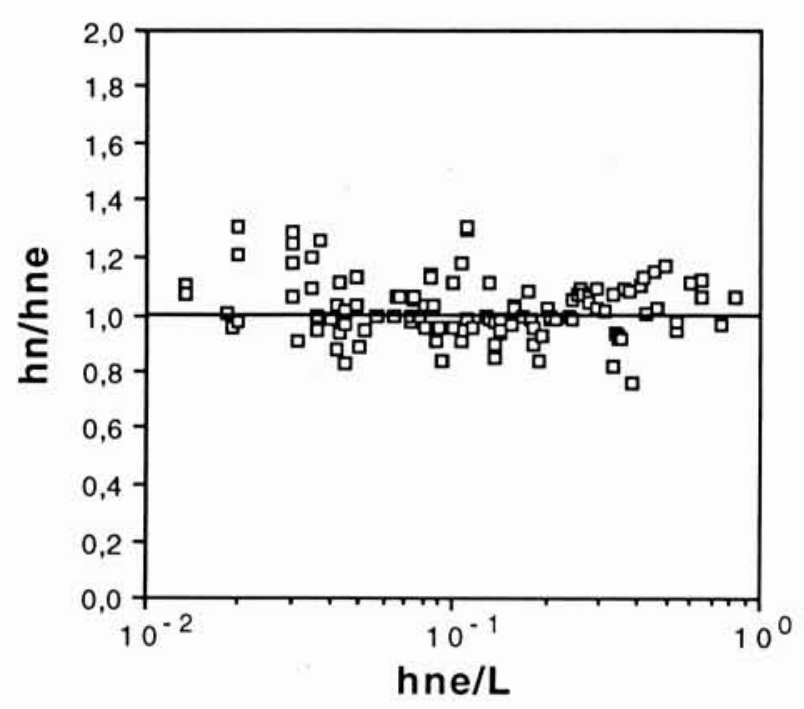

3. Comparaison hauteur normale expérimentale $\left(h_{u t}\right)$ - hauteur thérique $\left(h_{n}\right)$ (en utilisant la formule $\left.(10)\right)$ pour des écoulements uniformes en canal rectangulaire, en fonction du rapport $h_{m} / L|17|$.

Par exemple, sur la figure 3 , on compare les nouvelles prédictions théoriques en utilisant la formule (10) aux résultats expérimentaux. Il s'agit des mêmes résultats expérimentaux que ceux présentés sur la figure 2 . On constate que la formule (10) permet de redresser l'infléchissement de la courbe de la figure 2 observé pour des valeurs élevées de $h_{n} / L$.

\section{Régime graduellement varié}

Il a été montré récemment à la fois d'un point de vue théorique [18] et d'un point de vue expérimental [19], que les écoulements graduellement variés de laves torrentielles ont des caractéristiques analogues à celles que l'on observe en hydraulique à surface libre d'eau claire : courbes de remous, régimes torrentiel et fluvial, ressaut hydraulique. Pour cela on a supposé systématiquement, comme en hydraulique d'eau claire, que la contrainte moyenne à la paroi en une section quelconque d'un écoulement graduellement varié est égale à la contrainte moyenne à la paroi de l'écoulement uniforme ayant mêmes valeurs du débit et de la hauteur locale. En appliquant le théorème de conservation de la quantité de mouvement sur une tranche de fluide, il est alors possible de calculer la courbe de remous d'un écoulement donné dès que l'on connaît la contrainte à la paroi et la distribution des vitesses au sein du fluide (mais cette seconde condition est rarement remplie).

Dans le cas d'un plan infiniment large, si on néglige la non-uniformité des vitesses à travers une section, on obtient l'équation de la ligne d'eau suivante:

$$
\frac{\mathrm{d} h}{\mathrm{~d} x}=\frac{\sin i-\sin j(h, q)}{\cos i-\frac{U^{2}}{g h}}
$$

où $j(h, q)$ est approximativement égal à la pente qui permet d'avoir un écoulement uniforme à débit $q$ et avec

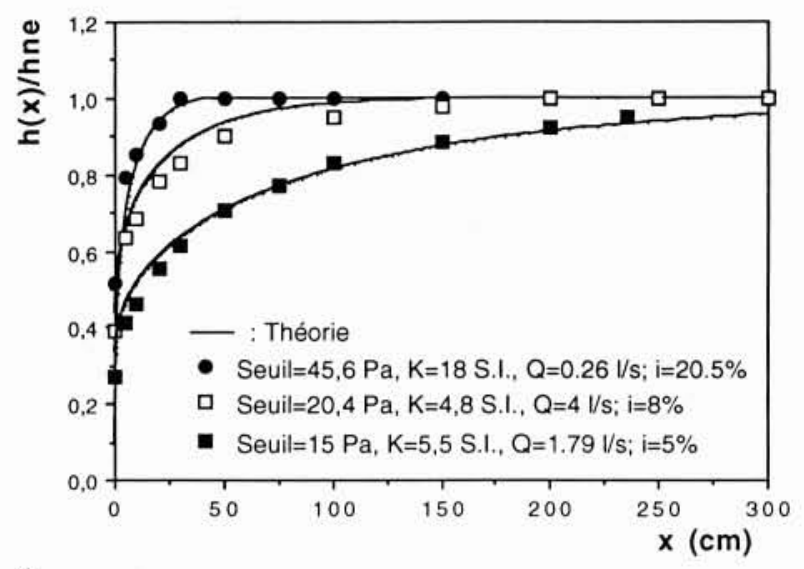

4. Exemples de courhes de remous observées expérimentalement avec différents mélanges boueux |19| et comparaison avec les prédictions théoriques.

une profondeur de fluide $h . j(h, q)$ peut donc être trouvé en égalisant la valeur de la contrainte à la paroi donnée $\operatorname{par}(8) \hat{a} \rho g h(\sin j)$. Des exemples de courbes de remous expérimentales comparées aux prédictions théoriques sont proposées figure 4. Dans le cas général (section quelconque) on peut aussi utiliser l'expression approximative (14), en calculant $j(h, q)$ en égalisant la valeur de contrainte normale donnée par (10) à $\rho g R(\sin j)$.

\section{Stabilité des écoulements}

Lorsque la pente ou le débit des écoulements deviennent trop grands, des vagues déferlantes peuvent se développer. Des ondes se déplacent à la surface dans le sens de l'écoulement et se transforment progressivement en vagues qui ont une hauteur et une vitesse plus grandes que les hauteurs et vitesses moyennes de l'écoulement uniforme théorique (cf. fig. 5). Ces vagues déferlantes sont aussi couramment observées lors d'écoulement d'eau claire de très faible profondeur sur des pentes assez fortes.

Pour les écoulements sur un plan incliné un critère de

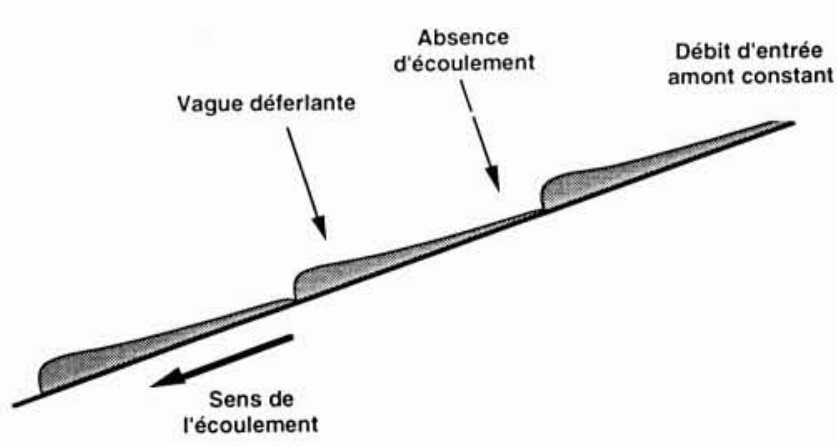

5. Aspect de profil des vagues déferlantes qui se produisent lors d'écoulements à des déhits ou des pentes élevés. 


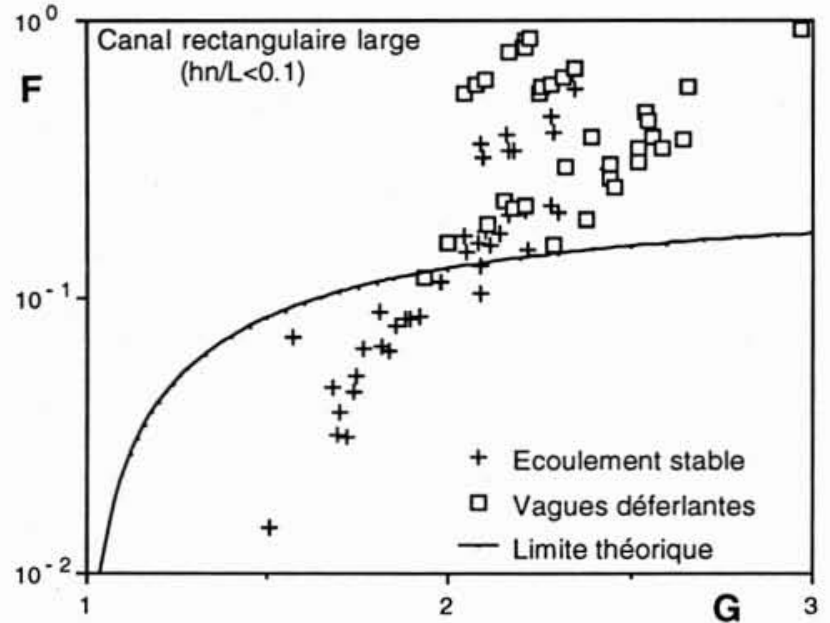

6. Différentes expériences d'écoulements avec alimentation amont constante de mélanges boueux. Comparaison expérience-théorie concernant la stabilité de ces écoulements.

stabilité peut être déduit de l'analyse uni-dimensionnelle de Trowbridge [20] :

$$
\sqrt{g h}\left(\frac{\partial \tau_{p}}{\partial U}\right)_{h}<\left(\tau_{p}-h\left(\frac{\partial \tau_{p}}{\partial h}\right)_{U}\right)
$$

où $U$ est la vitesse moyenne à travers une section. Ici encore il est particulièrement utile de disposer d'une expression de contrainte à la paroi du type (10). On peut en déduire aisément une expression simple du critère de stabilité de Trowbridge dans le cas d'un plan incliné infiniment large :

$$
F>\frac{4 G^{2}-3 G-1}{16 G^{2}+3 G+1} .
$$

Sur la figure 6 on a placé les résultats issus d'expériences d'écoulement de mélanges boueux dans un canal rectangulaire large $(h / L<0,1)$ ainsi que la courbe issue du critère (17). On peut voir que la théorie n'est pas parfaitement vérifiée mais cela peut être dû au fait que les instabilités n'avaient pas forcément le temps de se développer avant l'extrémité du canal, celui-ci n'étant pas infiniment long. On notera tout de même qu'aucun écoulement instable ne se produit en deçà de la courbe théorique. Pour des canaux de section différente le critère (17) pourrait être utilisé mais il est certainement plus approximatif parce que les hypothèses qui conduisent à cette expression ne sont plus vraies. Dans ce domaine, des études plus complètes, tenant notamment compte de la non-uniformité du profil des vitesses, devront être menées pour améliorer ces résultats. On peut au moins remarquer que, toutes choses égales par ailleurs, une instabilité se développe d'autant plus difficilement que le périmètre mouillé est grand [19].

D'un point de vue pratique, il est intéressant de constater que, d'après la formule (16), les laves boueuses donnent lieu à des écoulements par bouffées au moins lorsque le nombre de Froude est supérieur à 0,25 alors que pour l'eau ceci se produit lorsque $F$ est supérieur à 2 [21].

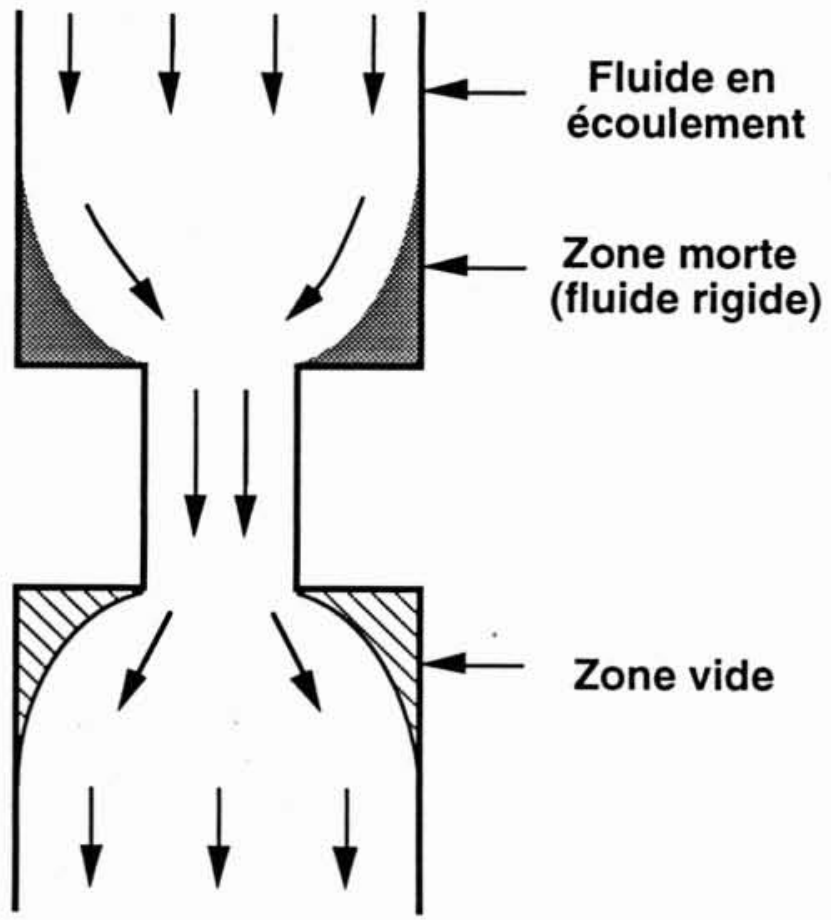

7. Aspect (vue de dessus) de l'écoulement d'un mélange boueux au passage d'un rétrécissement brusque puis d'un élargissement brusque.

\section{Singularités}

L'écoulement d'une lave torrentielle à travers une singularité du canal reste encore un problème à étudier en détail. Cependant, on sait déjà [22] que, contrairement à ce qui se passe avec de l'eau, il n'y a pas de pertes de charge importantes dues à de grands tourbillons autour de l'obstacle. En effet la lave subit surtout des déformations élongationnelles supplémentaires (par rapport au cisaillement nécessaire à l'avancement) qui lui permettent de s'écouler autour de l'obstacle. Les zones fortement turbulentes observées avec de l'eau claire sont en général remplacées ici par des zones de boue « mortes » (rigides) (cf. fig. 7). Ce phénomène est classiquement observé lors des écoulements de fluides à seuil au passage de singularités dans des conduites fermées. Il provient du fait que l'écoulement ne peut pas imposer une contrainte supérieure au seuil de contrainte, en tout point du fluide, notamment dans les parties les plus « retirées». Les zones où un fluide faiblement visqueux aurait une vitesse moyenne très faible sont alors remplacées par des zones rigides. On peut voir clairement ce phénomène lors d'un rétrécissement brusque (cf. fig. 7). Lors d'un élargissement brusque on trouve des zones « vides " que le fluide n'atteint qu'au bout d'un temps très long (cf. fig. 7).

\section{Références}

[1] Coussot P. (1994). - Rhéologie des laves torrentielles, La Houille Blanche (ce numéro). 
12] Coussot P. et PIAU J. M. (1993). - L'écoulement des boues. La Recherche, 24. n*258, pp. 1084-1091.

[3] Coussot P. (1992). - Rhéologie des laves torrentielles, thèse de Doctorat de I'Institut National Polytechnique de Grenoble. Grenoble. France, 420 p.

141 Coussot P. and PIAu J.-M. (1993). - A large-scale field coaxial cylinder rheometer to study the rheology of natural coarse suspensions. soumis au Journal of Rheology.

[5] HowARD C. D. D. (1963). - Flow of clay-water suspensions, Journal of the Hydraulic Division. Proceedings of the American society of Civil Engineers, HY5, pp. 89-97.

[6] PASl.AY P. R. and Slibar A. (1958). - Flow of an incompressible viscoplastic layer on an inclined plane, Transactions of the Society of Rheology, II, pp. 255-262.

171 Johnson A. M. (1970). - Physical processes in Geology, Freeman Cooper and Co, $577 \mathrm{p}$.

[8] De Kee D., Chнabra R. P., Powley M. B. and Roy S. (1990). - Flow of viscoplastic fluids on an inclined plane : evalution of vield stress, Chemical Engineering Communications. 96. pp. 229-239.

[9] KozICKI W. and TIU C. (1986). - Parametric modeling of flow geometries in non-Newtonian flows, in Flow dynamic and transport phenomena. Chapter 8, Encyclopedia of Fluid Mechanics, N. P. Cherimissinoff Editor, Gulf Publishing Company, Houston, London. Paris, Tokyo, pp. 199-252.

[10] WARD T. J. and O'BrIEN J. S. (1980), - Flume mechanics of mud flows, Completion report to National Science foundation Research Initiation, CER80-81TJW-JSO2, Fort Collins, Colorado, U.S.A., 130 p.

[11] ZhANG H. and REN Z. (1982). - Discussion on law of resistance of hyperconcentration flow in open channel, Scientia Sinica, A, $X X V, \mathrm{n}^{*} 12$, pp. 1332-1342.

112] NAIK B. (1983). - Mechanics of mudflow treated as the flow of a Bingham fluid. Ph. D. thesis, Washington State University, U.S.A.. 164 p.
113] Hanks R. W. (1986). - Principles of slurry pipeline hydraulics, in Slurry and suspension flow properties, Chapter 6. Encyclopedia of Fluid Mechanics, N. P. Cherimissinoff Editor, Gulf Publishing Company, Houston, London, Paris, Tokyo, pp. 213-276.

[14] WANG Z., LIN B., ZHANG X. (1993). - Instability of nonNewtonian open channel flow, E 1-23, Contributions to nonstationary sediment transport, W. Kron, I. H. W., Universität Karlsruhe, Germany.

[15] LAigl.e D. et Coussot P. (1993). - Modélisation numérique des écoulements de laves torrentielles, La Houille Blanche (ce numéro).

[16] Coussot P. et Laigl.e D. (1994). - Etude des laves torrentielles sur modèle réduit en similitude des phénomènes naturels. La Houille Blanche (ce numéro).

[17] Coussot P., Bossan E. (1993). - Steady, laminar, flow of concentrated mud suspensions in open channel. II Experiments and comparison with theory: uniform flows, soumis au Journal of Hydraulic Research.

[18] Coussot P. (1993), - Steady, laminar, flow of concentrated mud suspensions in open channel. I Theory, soumis au Journal of Hydraulic Research.

[19] Bossan E. (1993). - Hydraulique des laves torrentielles. DEA de mécanique, INPG. Grenoble.

[20] Trowbridge J. H. (1987). - Instability of concentrated free surface flows. Journal of Geophysical Research, 92 (C9), pp. 9523-9530.

[21] Dressler R. F. and Pohle F. V. (1953). - Resistance effects on hydraulic instability, Communications on Pure and Applied Mathematics, 6, pp. 93-96.

[22] Bossan E., Krage J.-U. et Tiberghien G. (1993). Hydraulique des laves torrentielles. Expériences d'écoulement à surface libre en canal ouvert. Projet de fin d'études de I'ENSHMG, Grenoble. France. 